\title{
ON THE EXTENSION OF QUASISYMMETRIC MAPS
}

\author{
Pekka Alestalo and Dmitry Alexandrovich Trotsenko \\ Aalto University School of Science, Department of Mathematics and Systems Analysis \\ PL 11100, FI-00076 Aalto, Finland; pekka.alestalo@aalto.fi \\ Sobolev Institute of Mathematics \\ Acad. Koptyug Avenue 4, 630090 Novosibirsk, Russia, and \\ Novosibirsk State University \\ Pirogova Street 2, 630090 Novosibirsk, Russia; trotsenk@yandex.ru
}

\begin{abstract}
We show that an $\varepsilon$-power-quasisymmetric map $f: A \rightarrow \mathbf{R}^{n}$ can be extended to a $C \varepsilon$-power-quasisymmetric map $F: \mathbf{R}^{n} \rightarrow \mathbf{R}^{n}$ if $A \subset \mathbf{R}^{n}$ satisfies a geometric thickness condition and $\varepsilon$ is small enough. The constant $C$ depends on $c$ and $n$ only.
\end{abstract}

\section{Introduction}

Let $A \subset \mathbf{R}^{n}$ and let $f: A \rightarrow \mathbf{R}^{n}$ be a mapping. A very general question is: Can $f$ be extended to a function $F: \mathbf{R}^{n} \rightarrow \mathbf{R}^{n}$ having similar properties as the original function $f$ ? This question has been studied and solved in many different cases. Classical results include the extension theorems for continuous (Tietze; or Brouwer, Lebesgue in this setting) and smooth (Whitney, $n=1$ ) functions $f$. In particular, the general Whitney extension problem " $F \in C^{m}\left(\mathbf{R}^{n}\right)$ ?" has been completely solved by Fefferman and his collaborators; cf. the Introduction and references in [DF].

However, in the case of continuous and injective $f$ there are still many open problems, if the extension is required to be a homeomorphism. Sometimes, extensions may not exist for topological reasons, but the most interesting cases arise when the (geo)metric properties of $A$ play a crucial role. Several results in the positive direction can be found, for example, in [AH, Jo, MS, Pa, PV, Re, Tr1, Vä]. The last reference also contains some basic counterexamples related to the quantitative properties of extension.

The present authors have studied this problem for $(1+\varepsilon)$-bilipschitz maps, and we present a similar result for quasisymmetric maps in this article. More precisely, it was proved by the authors and Väisälä in [ATV1] that $(1+\varepsilon)$-bilipschitz maps $f: A \rightarrow \mathbf{R}^{n}$ can be well approximated by isometries if the set $A$ satisfies a geometric condition related to its thickness. This result was applied in [ATV2] to show that, under similar conditions, the map $f$ has a $(1+C \varepsilon)$-bilipschitz extension $F: \mathbf{R}^{n} \rightarrow \mathbf{R}^{n}$. In [AT1], the present authors gave a geometric characterization for plane sets having this linear bilipschitz extension property. Note that here linear refers to the linear growth of the error term $C \varepsilon$, which is optimal.

Before stating our main theorem, we recall the definition of a quasisymmetric map.

doi:10.5186/aasfm.2016.4154

2010 Mathematics Subject Classification: Primary 30C65, 46B20.

Key words: Power quasisymmetric, extension, sturdy.

Acknowledgements: This cooperation was supported by the Vilho, Yrjö and Kalle Väisälä Fund, the Academy of Finland, and the Sobolev Institute of Mathematics. 
Definition 1.1. Let $X, Y$ be metric spaces with distance written as $|a-b|$, and let $\eta:[0, \infty) \rightarrow[0, \infty)$ be a homeomorphism, called a growth function. An embedding $f: X \rightarrow Y$ is $\eta$-quasisymmetric (abbr. $\eta$-QS) if

$$
\frac{|f(x)-f(y)|}{|f(x)-f(z)|} \leq \eta\left(\frac{|x-y|}{|x-z|}\right)
$$

for all $x, y, z \in X$ satisfying $x \neq z$. A function $f$ is quasisymmetric if it is $\eta$ quasisymmetric with some growth function $\eta$.

Remark 1.2. An $L$-bilipschitz map is $\eta$-quasisymmetric with $\eta(t)=L^{2} t$. Conversely, if the growth function $\eta$ is of the form $\eta(t)=C t$ for some constant $C$, then the corresponding map $f: X \rightarrow Y$ is a composition of a similarity and a bilipschitz map; see $[\mathrm{TuV}, 2.3]$.

It follows from $[\mathrm{TuV}, 3.12]$ and $[\mathrm{TrV}, 6.5]$ that every growth function $\eta$ can be replaced by a power form

$$
\eta(t)=C\left(t^{\alpha} \vee t^{1 / \alpha}\right)
$$

if the set $X$ is relatively connected, a class of spaces containing all connected ones, and even self-similar Cantor sets. Furthermore, this power-quasisymmetry property was completely characterized in [TrV]. In the present paper, we shall use these maps in the following case.

Definition 1.3. Let $\varepsilon>0$. An embedding $f: X \rightarrow Y$ is $\varepsilon$-power-quasisymmetric if it is $\eta$-QS with

$$
\eta(t)=(1+\varepsilon)\left(t^{1+\varepsilon} \vee t^{1 /(1+\varepsilon)}\right) .
$$

Examples of mappings satisfying this condition include quasiconformal maps with a small dilatation. In particular, suitable radial stretching maps provide examples that are not bilipschitz.

Another concept, called s-quasisymmetry, can also be used to study how close a mapping is to a similarity. We refer to [AT2] for details. However, it turns out that $s$-QS mappings are not suitable for extension results with sharp linear bounds, as in the following main theorem of the present article. See 2.2 for the definition of sturdiness.

Theorem 1.4. Let $A \subset \mathbf{R}^{n}$ be c-sturdy and let $f: A \rightarrow \mathbf{R}^{n}$ be $\varepsilon$-power-QS with $0<\varepsilon \leq \delta(c, n)$. Then $f$ has a $C \varepsilon$-power-QS extension $F: \mathbf{R}^{n} \rightarrow \mathbf{R}^{n}$, where $C=C(c, n)$.

\section{Preliminary results}

Our notation is standard and the same as in [AT1]. However, we recall the abbreviation $A(a, r)=A \cap \bar{B}(a, r)$ for a subset $A \subset \mathbf{R}^{n}$ and the following geometric properties of sets that are needed in our main result.

Definition 2.1. Thickness. For each unit vector $e \in \mathbf{S}^{n-1}$ we define the projection $\pi_{e}: \mathbf{R}^{n} \rightarrow \mathbf{R}$ by $\pi_{e} x=x \cdot e$. Let $A \neq \emptyset$ be a bounded set in $\mathbf{R}^{n}$, and let $d(A)$ denote the diameter of $A$. The thickness of $A$ is the number

$$
\theta(A)=\inf \left\{d\left(\pi_{e} A\right): e \in \mathbf{S}^{n-1}\right\} .
$$

Alternatively, $\theta(A)$ is the infimum of all $t>0$ such that $A$ lies between two parallel hyperplanes $F, F^{\prime}$ with mutual distance $d\left(F, F^{\prime}\right)=t$. We always have $0 \leq$ $\theta(A) \leq d(A)$. 
Definition 2.2. Sturdiness. Let $A \subset \mathbf{R}^{n}$. For $a \in A$ we set $s(a)=s_{A}(a)=$ $d(a, A \backslash\{a\})$, the distance from $a$ to the rest of $A$. Then $s(a)>0$ if and only if $a$ is isolated in $A$. Let $c \geq 1$. We say that the set $A \subset \mathbf{R}^{n}$ is $c$-sturdy if

(1) $\theta(A(a, r)) \geq 2 r / c$ whenever $a \in A, r \geq c s(a), A \not \subset B(a, r)$,

(2) $\theta(A) \geq d(\bar{A}) / c$.

If $A$ is unbounded, we omit (2), and the condition $A \not \subset B(a, r)$ of (1) is unnecessary.

Examples of sturdy sets in the plane include bounded Lipschitz-domains, $\mathbf{Z}^{2}$, and the snowflake curve. We recall the definition of a nearisometry from [ATV1, 1.1].

Definition 2.3. Let $X$ and $Y$ be metric spaces, let $f: X \rightarrow Y$, and let $\varepsilon>0$. We say that $f$ is an $\varepsilon$-nearisometry if

$$
|x-y|-\varepsilon \leq|f(x)-f(y)| \leq|x-y|+\varepsilon
$$

for all $x, y \in X$.

To shorten notation, we let a proper triple $T$ in a metric space $X$ consist of points $T=(x ; y, z)$ such that $y \neq x \neq z$, and define the ratio of $T$ as

$$
|T|=\frac{|x-y|}{|x-z|}
$$

An injective map $f: X \rightarrow Y$ maps each proper triple $T=(x ; y, z)$ in $X$ to another proper triple $T^{\prime}=(f(x) ; f(y), f(z))$ in $Y$.

We start with a couple of inequalities.

Lemma 2.4. Let $0<x \leq 1 \leq y$ and let $0 \leq \varepsilon \leq 1$. Then

a) $x^{-\varepsilon} \leq 1+\varepsilon(1-x) / x \leq 1+\varepsilon / x$;

b) $y^{\varepsilon} \leq 1+\varepsilon(y-1)$.

Proof. a) The mean value theorem, applied to $t \mapsto t^{\varepsilon}$ gives

$$
1-x^{\varepsilon}=\varepsilon t_{1}^{\varepsilon-1}(1-x) \leq \varepsilon x^{\varepsilon-1}(1-x),
$$

since $t_{1} \geq x$. Therefore

$$
x^{-\varepsilon}-1=\frac{1-x^{\varepsilon}}{x^{\varepsilon}} \leq \frac{\varepsilon(1-x)}{x},
$$

which implies the first inequality, and the second follows trivially.

b) This follows from the first part by substituting $x=1 / y$.

Lemma 2.5. Let $a, b>0$. Then

$$
\frac{a+t}{b-t} \leq \frac{a}{b}+2 t \frac{a+b}{b^{2}}
$$

for $0 \leq t \leq b / 2$. Moreover, if $a / b \leq 1$ and $c=4(a+b) / a b$, then

$$
\frac{a+t}{b-t} \leq(1+c t)\left(\frac{a}{b}\right)^{1 /(1+c t)}
$$

for $0 \leq t \leq b / 2$.

Proof. Let $g(t)=(a+t) /(b-t)$ and $h(t)=a / b+2 t(a+b) / b^{2}$. Then $g^{\prime \prime}(t)-h^{\prime \prime}(t)=$ $2(a+b) /(b-t)^{3} \geq 0$, so that $g-h$ is convex. Since $g(0)=h(0)$ and $g(b / 2)=h(b / 2)$, the first inequality follows.

For the second one, we estimate

$$
g^{\prime}(t)=\frac{a+b}{(b-t)^{2}} \leq \frac{4(a+b)}{b^{2}}
$$


for $t \leq b / 2$. On the other hand, for $f(t)=(1+c t)(a / b)^{1 /(1+c t)}$ we obtain

$$
\begin{aligned}
f^{\prime}(t) & =\frac{4(a+b)(a b+4 t(a+b)+a b \ln (b / a)}{a b(a b+4 t(a+b))}\left(\frac{a}{b}\right)^{a b /(a b+4 t(a+b))} \\
& \geq \frac{4(a+b)}{a b} \cdot \frac{a}{b}=\frac{4(a+b)}{b^{2}} \geq g^{\prime}(t),
\end{aligned}
$$

since the logarithmic term is nonnegative, the exponent is at most 1 , and $a / b \leq 1$. As $f(0)=g(0)=a / b$, we obtain $g(t) \leq f(t)$ for $0 \leq t \leq b / 2$, and the claim is proved.

Lemma 2.6. Let $0 \leq \varepsilon \leq 1$ and let $f: X \rightarrow Y$ be $\varepsilon$-power-QS. Suppose that there exist points $a, b \in X$ such that $|a-b|=d(X)=1$ and $|f(a)-f(b)|=1$. Then $f$ is a $23 \varepsilon$-nearisometry.

Proof. Let $x, y \in X, x \neq y$. To prove the nearisometry condition, we may assume that $|x-a| \geq 1 / 2$.

Let $T_{1}=(x ; y, a)$ and $T_{2}=(a ; x, b)$. Then $|x-y|=\left|T_{1}\right|\left|T_{2}\right|,|f(x)-f(y)|=$ $\left|T_{1}^{\prime}\right|\left|T_{2}^{\prime}\right|$

$$
\left|T_{1}\right|=\frac{|x-y|}{|x-a|} \leq 2 \quad \text { and } \quad\left|T_{2}\right|=\frac{|x-a|}{|a-b|} \leq 1
$$

We shall obtain the upper bound for $|f(x)-f(y)|$ by considering Cases 1 and 2 below, and the lower bound in Cases 3 and 4 after that.

Case 1. $\left|T_{1}\right| \leq 1$. Now we have

$$
\begin{aligned}
|f(x)-f(y)| & \leq(1+\varepsilon)^{2}\left|T_{1}\right|^{1 /(1+\varepsilon)}\left|T_{2}\right|^{1 /(1+\varepsilon)} \leq(1+3 \varepsilon)\left(\left|T_{1}\right|\left|T_{2}\right|\right)^{1-\varepsilon} \\
& =(1+3 \varepsilon)|x-y||x-y|^{-\varepsilon} \leq(1+3 \varepsilon)(|x-y|+\varepsilon) \leq|x-y|+7 \varepsilon
\end{aligned}
$$

by 2.4.a. obtain

Case $2.1 \leq\left|T_{1}\right| \leq 2$. Now $\left|T_{1}\right|^{2 \varepsilon} \leq 2 \varepsilon\left(\left|T_{1}\right|-1\right)+1 \leq 2 \varepsilon+1$ by 2.4 .b, and we

$$
\begin{aligned}
|f(x)-f(y)| & \leq(1+\varepsilon)^{2}\left|T_{1}\right|^{1+\varepsilon}\left|T_{2}\right|^{1 /(1+\varepsilon)} \leq(1+3 \varepsilon)\left|T_{1}\right|^{1+\varepsilon}\left|T_{2}\right|^{1-\varepsilon} \\
& =(1+3 \varepsilon)\left|T_{1}\right|^{2 \varepsilon}\left(\left|T_{1}\right|\left|T_{2}\right|\right)^{1-\varepsilon} \\
& \leq(1+3 \varepsilon)(2 \varepsilon+1)(|x-y|+\varepsilon) \leq|x-y|+23 \varepsilon,
\end{aligned}
$$

using 2.4.a again.

We have shown that $|f(x)-f(y)| \leq|x-y|+23 \varepsilon$ in both cases. It remains to show that $|f(x)-f(y)| \geq|x-y|-23 \varepsilon$ as well.

Let $T_{3}=(x ; a, y)$ and $T_{4}=(a ; b, x)$. Then $\left|T_{3}\right|\left|T_{4}\right|=1 /|x-y|,\left|T_{3}^{\prime}\right|\left|T_{4}^{\prime}\right|=$ $1 /|f(x)-f(y)|$,

$$
\left|T_{3}\right|=\frac{|x-a|}{|x-y|} \geq \frac{1}{2} \quad \text { and } \quad\left|T_{4}\right|=\frac{|a-b|}{|a-x|} \in[1,2] .
$$

Case 3. $\left|T_{3}\right| \leq 1$. Now $\left(1-\left|T_{3}\right|\right) /\left|T_{3}\right| \leq 2$ and $\left|T_{4}\right|-1 \leq 1$, and we get

$$
\begin{aligned}
\frac{1}{|f(x)-f(y)|} & =\left|T_{3}^{\prime}\right|\left|T_{4}^{\prime}\right| \leq(1+\varepsilon)^{2}\left|T_{3}\right|^{1-\varepsilon}\left|T_{4}\right|^{1+\varepsilon} \\
& \leq \frac{1+3 \varepsilon}{|x-y|}\left|T_{3}\right|^{-\varepsilon}\left|T_{4}\right|^{\varepsilon} \leq \frac{1+3 \varepsilon}{|x-y|}(1+2 \varepsilon)(1+\varepsilon) \leq \frac{1+23 \varepsilon}{|x-y|}
\end{aligned}
$$


by 2.4. Thus

$$
|f(x)-f(y)| \geq \frac{|x-y|}{1+23 \varepsilon} \geq(1-23 \varepsilon)|x-y| \geq|x-y|-23 \varepsilon,
$$

since $|x-y| \leq 1$.

Case 4. $\left|T_{3}\right| \geq 1$. Now $\left|T_{3}\right|\left|T_{4}\right| \geq 1$, so that using 2.4.b, we get

$$
\begin{aligned}
\frac{1}{|f(x)-f(y)|} & \leq(1+\varepsilon)^{2}\left|T_{3}\right|^{1+\varepsilon}\left|T_{4}\right|^{1+\varepsilon} \leq \frac{1+3 \varepsilon}{|x-y|}\left(1+\varepsilon\left(\left|T_{3}\right|\left|T_{4}\right|-1\right)\right) \\
& \leq \frac{1+3 \varepsilon}{|x-y|}\left(1+\frac{\varepsilon}{|x-y|}\right) .
\end{aligned}
$$

Thus

$$
\begin{aligned}
|f(x)-f(y)| & \geq \frac{|x-y|}{(1+3 \varepsilon)(1+\varepsilon /|x-y|)} \geq \frac{|x-y|}{1+7 \varepsilon /|x-y|} \\
& \geq|x-y|\left(1-\frac{7 \varepsilon}{|x-y|}\right)=|x-y|-7 \varepsilon .
\end{aligned}
$$

This completes the proof for the lower bound, and the lemma is proved.

\section{Approximation by similarities}

In this section we go through some preliminary results related to the question: How to approximate power-QS maps by similarities? The approximating similarities will be the main tool in constructing the extension needed for our main theorem.

Definition 3.1. For a similarity $S: \mathbf{R}^{n} \rightarrow \mathbf{R}^{n}$ let $\|S\|$ denote its similarity ratio.

Since $S$ is affine, this is also the norm of the corresponding linear transformation. When approximating a function $f: A \rightarrow \mathbf{R}^{n}$ with a similarity, we employ two equivalent ways to express the error of approximation:

$$
\|f-S\|_{A}=\sup \{|f(x)-S x| \mid x \in A\} \leq C\|S\| \varepsilon \Longleftrightarrow\left\|S^{-1} \circ f-\mathrm{id}\right\|_{A} \leq C \varepsilon .
$$

Theorem 3.2. Let $A \subset \mathbf{R}^{n}$ be compact and let $f: A \rightarrow l_{2}$ be $\varepsilon$-power-QS. Then there is a surjective similarity $S: l_{2} \rightarrow l_{2}$ such that $\left\|S^{-1} \circ f-\mathrm{id}\right\|_{A} \leq c_{n} d(A) \sqrt{\varepsilon}$. Also, we can choose $S$ so that $S \mathbf{R}^{n}=\mathbf{R}^{n}$.

Proof. Choose points $a, b \in A$ such that $|a-b|=d(A)$ and let $A_{0}=A / d(A)$, $M=|f(a)-f(b)|$. The map $g: A_{0} \rightarrow l_{2}$, defined by $g(x)=f(d(A) x) / M$, is $\varepsilon$-powerQS. From Lemma 2.6 it follows that $g$ is a $23 \varepsilon$-nearisometry. By Theorem [ATV1, 2.2], there is a surjective isometry $S_{0}: l_{2} \rightarrow l_{2}$ satisfying $\left\|S_{0}-g\right\|_{A_{0}} \leq c_{n} \sqrt{23 \varepsilon}$. Setting $S^{-1} x=d(A) S_{0}^{-1}(x / M)$ we obtain a surjective similarity $S: l_{2} \rightarrow l_{2}$ with $\left\|S^{-1} \circ f-\mathrm{id}\right\|_{A} \leq c_{n} \sqrt{23 \varepsilon} d(A)$.

In a similar way, using [ATV1, 3.3] instead of [ATV1, 2.2], we obtain the following result.

Theorem 3.3. Suppose that $c \geq 1$ and $A \subset \mathbf{R}^{n}$ is a compact set such that $\theta(A) \geq d(A) / c$. Let $f: A \rightarrow \mathbf{R}^{n}$ be $\varepsilon$-power-QS with $\varepsilon \leq 1$. Then there is a similarity $S: \mathbf{R}^{n} \rightarrow \mathbf{R}^{n}$ such that $\left\|S^{-1} \circ f-\mathrm{id}\right\|_{A} \leq c_{n} c \varepsilon d(A)$.

For easy reference, we note the following corollary to the preceding theorem.

Corollary 3.4. Let $A \subset \mathbf{R}^{n}$ be c-sturdy and let $f: A \rightarrow \mathbf{R}^{n}$ be $\varepsilon$-power-QS. Then for all $a \in A$ and $0<r \leq d(A)$, there is a similarity $S=S_{a, r}$ such that $S a=f(a)$ and $\|S-f\|_{A(a, r)} \leq c_{1}(c, n)\|S\| \varepsilon r$. 
Proof. Since $A$ is $c$-sturdy, we have

$$
\theta(A(a, r)) \geq 2 r / c \geq d(A(a, r)) / c .
$$

Thus 3.3 gives a similarity $S_{1}$ satisfying

$$
\left\|S_{1}-f\right\|_{A(a, r)} \leq 2\left\|S_{1}\right\| c c_{n} \varepsilon r .
$$

We claim that $S=S_{1}-S_{1} a+f(a)$ is the required similarity. If $x \in A(a, r)$, then

$$
|f(x)-S x| \leq\left|f(x)-S_{1} x\right|+\left|S_{1} a-f(a)\right| \leq 4\left\|S_{1}\right\| c c_{n} \varepsilon r .
$$

Since $\|S\|=\left\|S_{1}\right\|$, this proves the claim with $c_{1}(c, n)=4 c c_{n}$.

Definition 3.5. A similarity $S$ satisfying the properties of the preceeding corollary is called a $c_{1}$-special similarity for $(f, a, r)$.

Lemma 3.6. Let $X \subset \mathbf{R}^{n}$ be a bounded set with diameter $r=d(X)>0$, and let $f: X \rightarrow \mathbf{R}^{n}$ be a map that can be approximated by similarities $S_{1}$ and $S_{2}$ so that

$$
\left|S_{i}^{-1} f(x)-x\right| \leq \varepsilon r
$$

for all $x \in X, i=1,2$. If $0 \leq \varepsilon \leq 1 / 16$, then the similarity ratios satisfy the double inequality

$$
(1-8 \varepsilon)\left\|S_{2}\right\| \leq\left\|S_{2}\right\| /(1+8 \varepsilon) \leq\left\|S_{1}\right\| \leq(1+8 \varepsilon)\left\|S_{2}\right\| .
$$

Proof. Choose points $u, v \in A$ satisfying $|u-v|=r$, and write $x_{i}=S_{i}^{-1} f(u)$, $y_{i}=S_{i}^{-1} f(v)$. By assumption, we have $\left|x_{i}-u\right| \leq \varepsilon r$ and $\left|y_{i}-v\right| \leq \varepsilon r$ for $i=1,2$. Now

$$
\frac{\left\|S_{2}\right\|}{\left\|S_{1}\right\|}=\frac{\left\|S_{1}^{-1}\right\|}{\left\|S_{2}^{-1}\right\|}=\frac{\left|x_{1}-y_{1}\right|}{|f(u)-f(v)|} \frac{|f(u)-f(v)|}{\left|x_{2}-y_{2}\right|}=\frac{\left|x_{1}-y_{1}\right|}{\left|x_{2}-y_{2}\right|} \leq \frac{r+2 \varepsilon r}{r-2 \varepsilon r} \leq 1+8 \varepsilon
$$

by 2.5. This proves the second inequality, and the last one follows by interchanging $S_{1}$ and $S_{2}$.

The following result was essentially proved by the second author in [Tr2], but in a somewhat different context. We therefore give a reformulation which is better suited for our needs, and a complete proof. See also [Vä, 3.9].

Theorem 3.7. Let $0 \leq \varepsilon \leq 1 / 100$ and let $F: \mathbf{R}^{n} \rightarrow \mathbf{R}^{n}$ be a mapping having the following approximation property: For every $x \in \mathbf{R}^{n}$ and $r>0$, there is a similarity $S=S_{x, r}$ of $\mathbf{R}^{n}$ satisfying

$$
\|S \circ F-\mathrm{id}\|_{\bar{B}(x, r)} \leq \varepsilon r .
$$

Then $f$ is $50 \varepsilon$-power-quasisymmetric.

Proof. Let $x, y, z \in \mathbf{R}^{n}$ be distinct points, and let $T=(x ; y, z), T^{\prime}=(f(x) ; f(y)$, $f(z))$. We shall prove that

$$
(1-25 \varepsilon)|T|^{1+12 \varepsilon} \leq\left|T^{\prime}\right| \leq(1+25 \varepsilon)|T|^{1-16 \varepsilon}
$$

in the case where $|x-y| \leq|x-z|$, i.e. $|T| \leq 1$. Because of the double inequality (3.1), the case $|T| \geq 1$ follows by interchanging $y$ and $z$, and using $1 /(1+2 a) \leq$ $1-a \leq 1 /(1+a)$.

We thus assume that $|x-y| \leq|x-z|=r$, and then $y, z \in \bar{B}(x, r)$. Let $B_{i}=\bar{B}\left(x, r / 2^{i}\right)$ and choose an integer $k$ such that

$$
\frac{r}{2^{k+1}}<|x-y| \leq \frac{r}{2^{k}}
$$


For every $0 \leq i<k$, there is a similarity $S_{i}$ such that

$$
\left\|S_{i} f-\mathrm{id}\right\|_{B_{i}} \leq \frac{\varepsilon r}{2^{i}} \quad \text { and } \quad\left\|S_{i+1} f-\mathrm{id}\right\|_{B_{i+1}} \leq \frac{\varepsilon r}{2^{i+1}} .
$$

Thus $\left\|S_{j} f-\mathrm{id}\right\|_{B_{i+1}} \leq 2 \varepsilon \cdot r / 2^{i+1}=\varepsilon d\left(B_{i+1}\right)$ for $j=i$ and $j=i+1$. Applying lemma 3.6 to the successive balls $B_{i}$ and $B_{i+1}$, it follows that

$$
(1-8 \varepsilon)^{k}\left\|S_{0}\right\| \leq\left\|S_{k}\right\| \leq(1+8 \varepsilon)^{k}\left\|S_{0}\right\| .
$$

By construction, we have $k \leq \log _{2}(1 /|T|)<k+1$. Therefore

$$
(1+8 \varepsilon)^{k} \leq|T|^{-12 \varepsilon}
$$

using the inequality $(1+t)^{\log _{2} s} \leq s^{1.5 t}$, which is valid for $s \geq 1$ and $0 \leq t \leq 1 / 2$. Similarly,

$$
(1-8 \varepsilon)^{k} \geq|T|^{16 \varepsilon}
$$

using the inequality $(1-t)^{\log _{2} s} \geq s^{-2 t}$, valid for $s \geq 1$ and $0 \leq t \leq 1 / 2$. Combining the above inequalities, we obtain the estimates

$$
|T|^{16 \varepsilon}\left\|S_{0}\right\| \leq\left\|S_{k}\right\| \leq|T|^{-12 \varepsilon}\left\|S_{0}\right\| .
$$

We are now ready to estimate $|f(x)-f(y)|$ and $|f(x)-f(z)|$ separately, starting from the first expression. Since $\left|S_{k} f(y)-y\right| \leq \varepsilon r / 2^{k}$, we first obtain

$$
|x-y|-\frac{\varepsilon r}{2^{k}} \leq\left|S_{k} f(y)-x\right| \leq|x-y|+\frac{\varepsilon r}{2^{k}} .
$$

Using this and $\left|S_{k} f(x)-x\right| \leq \varepsilon r / 2^{k} \leq 2 \varepsilon|x-y|$, we have

$$
(1-4 \varepsilon)|x-y| \leq\left|S_{k} f(x)-S_{k} f(y)\right| \leq(1+4 \varepsilon)|x-y| .
$$

Combining this with the equation

$$
\left\|S_{k}\right\|=\frac{\left|S_{k} f(x)-S_{k} f(y)\right|}{|f(x)-f(y)|}
$$

and with (3.2), we get

$$
\frac{(1-4 \varepsilon)|x-y|}{|T|^{-12 \varepsilon}\left\|S_{0}\right\|} \leq|f(x)-f(y)| \leq \frac{(1+4 \varepsilon)|x-y|}{|T|^{16 \varepsilon}\left\|S_{0}\right\|} .
$$

Next we estimate $|f(x)-f(z)|$. Using the approximation $S_{0}$ for both $f(x)$ and $f(z)$, we obtain

$$
(1-2 \varepsilon)|x-z| \leq\left|S_{0} f(x)-S_{0} f(z)\right| \leq(1+2 \varepsilon)|x-z| .
$$

From this we get the double inequality

$$
\frac{(1-2 \varepsilon)|x-z|}{|f(x)-f(z)|} \leq\left\|S_{0}\right\|=\frac{\left|S_{0} f(x)-S_{0} f(z)\right|}{|f(x)-f(z)|} \leq \frac{(1+2 \varepsilon)|x-z|}{|f(x)-f(z)|} .
$$

Combining these estimates for $\left\|S_{0}\right\|$ with (3.3), we obtain the double inequality

$$
\frac{(1-4 \varepsilon)|x-y|}{|T|^{-12 \varepsilon}(1+2 \varepsilon)|x-z|} \leq \frac{|f(x)-f(y)|}{|f(x)-f(z)|} \leq \frac{(1+4 \varepsilon)|x-y|}{|T|^{16 \varepsilon}(1-2 \varepsilon)|x-z|} .
$$

From this, the estimates (3.1) easily follow, and the proof is complete.

Definition 3.8. Suppose that $A \subset \mathbf{R}^{n}, a \in A, r>0$, and $c \geq 1$. We say that an $n$-simplex $\Delta$ is $c$-special for $(A, a, r)$, or briefly a $c$-special simplex of $A$, if

(1) $\Delta^{0} \subset A(a, r)$, and

(2) the smallest height $b(\Delta)$ of $\Delta$ satisfies $b(\Delta) \geq r / c$. 
Since $d(\Delta) \leq 2 r$, we have $\rho(\Delta)=d(\Delta) / b(\Delta) \leq 2 c$ for every $c$-special simplex $\Delta$ of $A$.

Lemma 3.9. Let $A \subset \mathbf{R}^{n}$ be closed, unbounded, and c-sturdy. If $a \in A$ and $r \geq c s(a)$, then there is a $c$-special simplex for $(A, a, r)$.

Proof. See [ATV2, 3.6].

The following lemma is almost identical with [ATV2, 3.12], and we do not repeat the proof.

Lemma 3.10. Let $A \subset \mathbf{R}^{n}$ be closed, unbounded, and c-sturdy, and let $f: A \rightarrow$ $\mathbf{R}^{n}$ be $(1+\varepsilon)$-power-QS. Suppose that $a, b \in A$ and $r_{1} \geq c s(a), r_{2} \geq c s(b)$. If $S$ and $T$ are $c_{1}$-special similarities for $\left(f, a, r_{1}\right)$ and $\left(f, b, r_{2}\right)$, respectively, then they have the same orientation, provided that $0 \leq \varepsilon \leq \delta(c, n)$.

Lemma 3.11. Let $\Delta \subset \mathbf{R}^{n}$ be an $n$-simplex, and let $S, T: \Delta \rightarrow \mathbf{R}^{n}$ be similarities such that $\|S-T\|_{\Delta^{0}} \leq \eta$. Then $|\|S\|-\|T\|| \leq 2 \eta / d(\Delta)$ and

$$
|S x-T x| \leq \eta(1+M|x-v| / d(\Delta))
$$

for all $x \in \Delta$ and $v \in \Delta^{0}$, where $M=4+6 n \rho(\Delta)(1+\rho(\Delta))^{n-1}$.

Proof. See [Vä, 2.11].

\section{Proof of the main theorem}

The following result reduces the extension problem to the case of unbounded sturdy sets. This makes it easier to handle the definition of sturdiness, because Condition 2.2(2) can be omitted.

Theorem 4.1. Suppose that all unbounded c-sturdy sets $A \subset \mathbf{R}^{n}$ have the following property: There is $\delta=\delta(c, n)>0$ such that every $\varepsilon$-power-QS map $f: A \rightarrow$ $\mathbf{R}^{n}$ with $0 \leq \varepsilon \leq \delta$ extends to a $C \varepsilon$-power-QS map $F: \mathbf{R}^{n} \rightarrow \mathbf{R}^{n}$, where $C=$ $C(c, n)$. Then all c-sturdy sets $A \subset \mathbf{R}^{n}$ have the same property with $\delta$ replaced by $\delta^{\prime}=\delta(6 c, n) / 34 c_{n} c$ and $C$ replaced by $\left.C^{\prime}=34 c_{n} c C(6 c, n)\right)$. Here $c_{n}$ is the constant from 3.3.

Proof. Suppose that $A \subset \mathbf{R}^{n}$ is bounded and $c$-sturdy. Let $\varepsilon \leq \delta^{\prime}(c, n)$ and let $f: A \rightarrow \mathbf{R}^{n}$ be $\varepsilon$-power-quasisymmetric. Setting $R=d(A)$, we have $\theta(A) \geq R / c$ by sturdiness. By 3.3, there is a similarity $S: \mathbf{R}^{n} \rightarrow \mathbf{R}^{n}$ such that $\|S \circ f-\mathrm{id}\|_{A} \leq c_{n} c \varepsilon R$.

We may assume that $0 \in A$, so that $A \subset \bar{B}(R)$. Let $A_{1}=A \cup\left(\mathbf{R}^{n} \backslash B(2 R)\right)$. Then it follows from [ATV2, 4.1] that $A_{1}$ is $6 c$-sturdy. We extend $f$ to a map $f_{1}: A_{1} \rightarrow \mathbf{R}^{n}$ by setting $f_{1}(x)=S^{-1}(x)$ in case $|x| \geq 2 R$.

We shall prove below that $f_{1}$ is $34 c_{n} c \varepsilon$-power-quasisymmetric. Since $A_{1}$ is unbounded and $6 c$-sturdy, the assumptions give a $C^{\prime} \varepsilon$-power-quasisymmetric extension $F: \mathbf{R}^{n} \rightarrow \mathbf{R}^{n}$ of $f_{1}$. This will be the required extension of $f$ also.

It remains to prove that $f_{1}$ is $34 c_{n} c \varepsilon$-power-quasisymmetric. Let thus $x, y, z \in A_{1}$ be distinct points and let $T=(x ; y, z)$. We divide the proof into six nontrivial cases. Let $\sigma=\left\|S^{-1}\right\|$ be the similarity ratio of $S^{-1}$.

Case 1. $x \in A, y, z \notin A$. Using the approximation $S^{-1}$, we obtain

$$
\left|f(x)-S^{-1}(y)\right|=\sigma|S f(x)-y| \leq \sigma(|S f(x)-x|+|x-y|) \leq \sigma\left(|x-y|+c_{n} c \varepsilon R\right) .
$$


We shall use similar estimates quite often, and also in the opposite direction in the form $\left|f(x)-S^{-1}(z)\right| \geq \sigma\left(|x-y|-c_{n} c \varepsilon R\right)$. Thus in this case

$$
\left|T^{\prime}\right|=\frac{\left|f(x)-S^{-1}(y)\right|}{\left|f(x)-S^{-1}(z)\right|}=\frac{|S f(x)-y|}{|S f(x)-z|} \leq \frac{|x-y|+c_{n} c \varepsilon R}{|x-z|-c_{n} c \varepsilon R} .
$$

If $|x-z| \leq|x-y|$ and $\varepsilon \leq 1 / 2 c_{n} c$, then the first part of 2.5 gives

$$
\begin{aligned}
\left|T^{\prime}\right| & \leq|T|+2 c_{n} c \varepsilon R \frac{|x-y|+|x-z|}{|x-z|^{2}} \\
& \leq|T|+4 c_{n} c \varepsilon R \frac{|x-y|}{|x-z|^{2}} \leq\left(1+4 c_{n} c \varepsilon\right)|T|,
\end{aligned}
$$

since $|x-z| \geq R$. If $|x-y| \leq|x-z|$, then $|T| \leq 1$ and the second part of 2.5 gives

$$
\left|T^{\prime}\right| \leq\left(1+M c_{n} c \varepsilon R\right)|T|^{1 /\left(1+M c_{n} c \varepsilon R\right)} .
$$

Here

$$
M c_{n} c \varepsilon R=4 c_{n} c \varepsilon R \frac{|x-y|+|x-z|}{|x-y||x-z|} \leq 8 c_{n} c \varepsilon
$$

since $|x-y| \geq R$. This completes the proof of Case 1 .

Case 2. $y \in A, x, z \notin A$. In this case, we have

$$
\left|T^{\prime}\right|=\frac{S^{-1}(x)-f(y)}{\left|S^{-1}(x)-S^{-1}(z)\right|}=\frac{|x-S f(y)|}{|x-z|} \leq \frac{|x-y|+c_{n} c \varepsilon R}{|x-z|} \leq\left(1+c_{n} c \varepsilon\right)|T|,
$$

since $R \leq|x-y|$. Case 2 is thus proved.

Case 3. $z \in A, x, y \notin A$. In this case $|x-z| \geq R$, and we have

$$
\begin{aligned}
\left|T^{\prime}\right| & =\frac{\left|S^{-1}(x)-S^{-1}(y)\right|}{\left|S^{-1}(x)-f(z)\right|}=\frac{|x-y|}{|x-S f(z)|} \leq \frac{|x-y|}{|x-z|-c_{n} c \varepsilon R} \\
& \leq \frac{|x-y|}{|x-z|}\left(1+\frac{2 c_{n} c \varepsilon R}{|x-z|}\right) \leq\left(1+2 c_{n} c \varepsilon\right) \frac{|x-y|}{|x-z|}
\end{aligned}
$$

if $\varepsilon \leq 1 / 2 c_{n} c$. Case 3 is now proved.

Case 4. $y, z \in A, x \notin A$. Now

$$
\left|T^{\prime}\right|=\frac{|x-S f(y)|}{|x-S f(z)|} \leq \frac{|x-y|+c_{n} c \varepsilon R}{|x-z|-c_{n} c \varepsilon R} .
$$

From this, the proof goes on as in Case 1 with two subcases, since $|x-y| \geq R$ and $|x-z| \geq R$ also here.

Case 5. $x, z \in A, y \notin A$. We have

$$
\left|T^{\prime}\right|=\frac{\left|f(x)-S^{-1}(y)\right|}{|f(x)-f(z)|}=\frac{\sigma|S f(x)-y|}{|f(x)-f(z)|} .
$$

Choose $w \in A$ such that $|x-w|$ is maximal. Then $R / 2 \leq|x-w| \leq R$. Now

$$
|f(x)-f(w)|=\sigma|S f(x)-S f(w)| \geq \sigma\left(|x-w|-2 c_{n} c \varepsilon R\right),
$$

and since

$$
\frac{|f(x)-f(w)|}{|f(x)-f(z)|} \leq(1+\varepsilon)\left(\frac{|x-w|}{|x-z|}\right)^{1+\varepsilon}
$$


we obtain

$$
\begin{aligned}
\frac{1}{|f(x)-f(z)|} & \leq(1+\varepsilon)\left(\frac{|x-w|}{|x-z|}\right)^{\varepsilon} \frac{1}{\sigma\left(1-2 c_{n} c \varepsilon R /|x-w|\right)} \cdot \frac{1}{|x-z|} \\
& \leq(1+\varepsilon)\left(1+8 c_{n} c \varepsilon\right)\left(\frac{|x-y|}{|x-z|}\right)^{\varepsilon} \frac{1}{\sigma|x-z|}
\end{aligned}
$$

where we used $|x-w|^{\varepsilon} \leq|x-y|^{\varepsilon}$ and $R \leq 2|x-w|$.

Combining these estimates and using $R \leq|x-y|$, we get

$$
\begin{aligned}
\left|T^{\prime}\right| & \leq \frac{|x-y|+c_{n} c \varepsilon R}{|x-z|}(1+\varepsilon)\left(1+8 c_{n} c \varepsilon\right)\left(\frac{|x-y|}{|x-z|}\right)^{\varepsilon} \\
& \leq\left(1+c_{n} c \varepsilon\right)(1+\varepsilon)\left(1+8 c_{n} c \varepsilon\right)\left(\frac{|x-y|}{|x-z|}\right)^{1+\varepsilon} \leq\left(1+34 c_{n} c \varepsilon\right)\left(\frac{|x-y|}{|x-z|}\right)^{1+\varepsilon} .
\end{aligned}
$$

This completes the proof of case 5 .

Case 6. $x, y \in A, z \notin A$. We have $|x-y| \leq R \leq|x-z|$ in this case. Choose again $w \in A$ such that $|w-x|$ is maximal; thus $R / 2 \leq|x-w| \leq R$ and $|x-y| /|x-w| \leq 2$.

Subcase 6 a. $1 \leq|x-y| /|x-w| \leq 2$. Now

$$
|f(x)-f(w)| \leq \sigma\left(|x-w|+2 c_{n} c \varepsilon R\right)
$$

and

$$
\left(\frac{|x-y|}{|x-w|}\right)^{1+\varepsilon} \leq 2^{\varepsilon} \frac{|x-y|}{|x-w|} \leq(1+\varepsilon) \frac{|x-y|}{|x-w|} .
$$

Using $\varepsilon$-power-quasisymmetry, we obtain

$$
\begin{aligned}
|f(x)-f(y)| & \leq(1+\varepsilon)\left(\frac{|x-y|}{|x-w|}\right)^{1+\varepsilon}|f(x)-f(w)| \\
& \leq(1+\varepsilon)^{2} \sigma\left(|x-w|+2 c_{n} c \varepsilon R\right) \frac{|x-y|}{|x-w|} \\
& \leq(1+\varepsilon)^{2} \sigma\left(1+2 c_{n} c \varepsilon\right)|x-y| \leq\left(1+7 c_{n} c \varepsilon\right) \sigma|x-y| .
\end{aligned}
$$

Therefore, we get

$$
\begin{aligned}
\left|T^{\prime}\right| & =\frac{|f(x)-f(y)|}{\left|f(x)-S^{-1}(z)\right|} \leq \frac{\left(1+7 c_{n} c \varepsilon\right) \sigma|x-y|}{\sigma\left(|x-z|-c_{n} c \varepsilon R\right)} \\
& \leq\left(1+7 c_{n} c \varepsilon\right)\left(1+2 c_{n} c \varepsilon\right) \frac{|x-y|}{|x-z|} \leq\left(1+23 c_{n} c \varepsilon\right)|T|
\end{aligned}
$$

Subcase 6b. $|x-y| \leq|x-w|$. We write $1-\varepsilon^{\prime}=1 /(1+\varepsilon)$ to simplify notation, and then

$$
\frac{|f(x)-f(y)|}{|f(x)-f(w)|} \leq(1+\varepsilon)\left(\frac{|x-y|}{|x-w|}\right)^{1-\varepsilon^{\prime}} .
$$

Here

$$
|f(x)-f(w)| \leq \sigma\left(|x-w|+2 c_{n} c \varepsilon R\right)
$$

and

$$
\left|f(x)-S^{-1}(z)\right| \geq \sigma\left(|x-z|-c_{n} c \varepsilon R\right) .
$$


Combining these estimates and using $|x-w|^{\varepsilon^{\prime}} \leq|x-z|^{\varepsilon^{\prime}}$, we get

$$
\begin{aligned}
\left|T^{\prime}\right| & \leq(1+\varepsilon) \frac{|x-y|}{|x-w|} \frac{|x-w|^{\varepsilon^{\prime}}}{|x-y|^{\prime}} \frac{|x-w|+2 c_{n} c \varepsilon R}{|x-z|-c_{n} c \varepsilon R} \\
& \leq(1+\varepsilon) \frac{|x-y|}{|x-w|} \frac{|x-z|^{\varepsilon^{\prime}}}{|x-y|^{\varepsilon^{\prime}}} \frac{1+2 c_{n} c \varepsilon}{1-c_{n} c \varepsilon} \\
& \leq\left(1+18 c_{n} c \varepsilon\right) \frac{|x-y|^{1-\varepsilon^{\prime}}}{|x-z|^{1-\varepsilon^{\prime}}} \leq\left(1+18 c_{n} c \varepsilon\right)\left(\frac{|x-y|}{|x-z|}\right)^{1 /(1+\varepsilon)} .
\end{aligned}
$$

This completes the proof of Subcase $6 \mathrm{~b}$.

Finally, comparing the constants and restrictions obtained in different cases, we obtain the expressions for $\delta^{\prime}$ and $C^{\prime}$ given in the theorem.

Proof of the main theorem 1.4. The proof will be carried out in several steps, some of which are similar to the ones used in the proof of [ATV2, Section 4]. We give here an essentially complete proof, but some technical details that can be found in the above-mentioned article are omitted. Also, some very similar cases and subcases are compressed in the last part of the proof.

By 4.1 we may assume that $A \subset \mathbf{R}^{n}$ is closed, unbounded, and $c$-sturdy, and $f: A \rightarrow \mathbf{R}^{n}$ is $\varepsilon$-power-QS. Let $K$ be a decomposition of $G=\mathbf{R}^{n} \backslash A$ into Whitney cubes $Q$ such that

(i) $1 \leq d(Q, A) / d(Q)<3$;

(ii) $1 / 2 \leq d(Q) / d\left(Q^{\prime}\right) \leq 2$ if $Q, Q^{\prime} \in K$ and $Q \cap Q^{\prime} \neq \emptyset$.

We first define the extension $F$ in the set $K^{0}$ in the vertices of the cubes $Q \in K$. Let $v \in K^{0}$ and choose a point $a_{v} \in A$ such that $r_{v}=\left|v-a_{v}\right|=d(v, A)$. Let $t_{v}=s\left(a_{v}\right) \vee 8 r_{v}$. By 3.5 there is a $C_{0}$-special similarity $S_{v}$ for $\left(f, a_{v}, c t_{v}\right)$ satisfying $S_{v} a_{v}=f\left(a_{v}\right)$ and

$$
\left\|S_{v}-f\right\|_{A\left(a_{v}, c t_{v}\right)} \leq C_{0}\left\|S_{v}\right\| t_{v} \varepsilon
$$

where $C_{0}=4 c^{2} c_{n}$. We choose $S_{u}=S_{v}$ whenever $a_{u}=a_{v}=a$ and $u, v \in \bar{B}(a, s(a) / 8)$. Furthermore, by 3.10 we may assume that all these similarities have positive orientation. We define $F(v)=S_{v} v$.

Next we triangulate each $Q \in K$ in a standard way to obtain a collection $W$ of $n$-simplexes $W=\{\Delta \in Q \mid Q \in K\}$. These simplexes satisfy

$$
d(\Delta)=\lambda \sqrt{n}, \quad b(\Delta)=\lambda / \sqrt{2}, \quad \rho(\Delta)=\sqrt{2 n},
$$

if $\Delta \in Q$ and $Q$ has sides of length $2 \lambda$.

After this, we extend $F$ to each $\Delta \in W$ in an affine way. Setting $F \mid A=f$, we obtain a map $F: \mathbf{R}^{n} \rightarrow \mathbf{R}^{n}$ that extends $f$.

Fact 1. Let $Q \in K$ and $u, v \in Q \cap K^{0}$. Then

(i) $\left|a_{u}-a_{v}\right| \leq 3\left(r_{u} \vee r_{v}\right)$;

(ii) $r_{v} \leq 2 r_{u}$;

(iii) $\left\|S_{u}-S_{v}\right\|_{Q} \leq C_{1}\left(\left\|S_{u}\right\| \vee\left\|S_{v}\right\|\right)\left(r_{u} \vee r_{v}\right) \varepsilon$;

(iv) $t_{u} \leq 2 t_{v}$ and $\bar{B}\left(a_{u}, t_{u}\right) \subset \bar{B}\left(a_{v}, 3 t_{v}\right)$;

(v) $\left(1-16 C_{0} \varepsilon\right)\left\|S_{v}\right\| \leq\left\|S_{u}\right\| \leq\left(1+16 C_{0} \varepsilon\right)\left\|S_{v}\right\|$.

Proof. The first two inequalities are the same as in [ATV2, p. 965]. To prove (iii) we may assume that $r_{u} \leq r_{v}$. 
If $t_{v}=s\left(a_{v}\right) \geq 8 r_{v}$, then $a_{v}$ is isolated in $A$. We claim that $a_{u}=a_{v}$ in this case. If not, then (i) and (ii) imply

$$
s\left(a_{v}\right) \leq\left|a_{u}-a_{v}\right| \leq 3 r_{v} \leq 3 s\left(a_{v}\right) / 8,
$$

a contradiction. Thus $a_{u}=a_{v}$, and since $r_{u} \leq r_{v} \leq 3 s\left(a_{v}\right) / 8$, we have $S_{u}=S_{v}$. Therefore, (iii) is trivially true in this case.

We now assume that $t_{v}=8 r_{v} \geq s\left(a_{v}\right)$.

Case 1. $r_{u} \geq \operatorname{cs}\left(a_{u}\right)$. Now $t_{u}=8 r_{u}$ and $r_{v} \geq r_{u} \geq c s\left(a_{u}\right)$. By 3.9 there is a $c$-special simplex $\Delta_{u}$ for $\left(A, a_{u}, r_{v}\right)$ satisfying

$$
\Delta_{u}^{0} \subset A\left(a_{u}, r_{v}\right), \quad b\left(\Delta_{u}\right) \geq r_{v} / c, \rho\left(\Delta_{u}\right) \leq 2 c .
$$

Since $r_{v} \leq 2 r_{u}<t_{u}$ by (ii), we obtain by (4.1) the inequality

$$
\left\|S_{u}-f\right\|_{\Delta_{u}^{0}} \leq C_{0}\left\|S_{u}\right\| \varepsilon t_{u} \leq C_{0}\left\|S_{u}\right\| \varepsilon t_{v} .
$$

By (i), we also have $\bar{B}\left(a_{u}, r_{v}\right) \subset \bar{B}\left(a_{v}, 4 r_{v}\right)$, and thus (4.1) implies that

$$
\left\|S_{v}-f\right\|_{\Delta_{u}^{0}} \leq C_{0} \varepsilon t_{v}\left\|S_{v}\right\| .
$$

Consequently,

$$
\left\|S_{u}-S_{v}\right\|_{\Delta_{u}^{0}} \leq 2 C_{0} \varepsilon t_{v}\left(\left\|S_{u}\right\| \vee\left\|S_{v}\right\|\right)
$$

and 3.11 implies that

$$
\begin{aligned}
\left|\left\|S_{u}\right\|-\left\|S_{v}\right\|\right| & \leq 4 C_{0} \varepsilon t_{v}\left(\left\|S_{u}\right\| \vee\left\|S_{v}\right\|\right) / d\left(\Delta_{u}\right)=32 C_{0} \varepsilon r_{v}\left(\left\|S_{u}\right\| \vee\left\|S_{v}\right\|\right) / d\left(\Delta_{v}\right) \\
& \leq 192 C_{0} \varepsilon\left(\left\|S_{u}\right\| \vee\left\|S_{v}\right\|\right) .
\end{aligned}
$$

Let $x \in Q$. Choose a vertex $z \in \Delta_{u}^{0}$ and apply 3.11 to get

$$
\left|S_{u} x-S_{v} x\right| \leq 2 C_{0} \varepsilon t_{v}\left(1+M|x-z| / d\left(\Delta_{u}\right)\right)\left(\left\|S_{u}\right\| \vee\left\|S_{v}\right\|\right) .
$$

Here

$$
M=4+6 n \rho\left(\Delta_{u}\right)\left(1+\rho\left(\Delta_{u}\right)\right)^{n-1} \leq 4+12 n(1+2 c)^{n-1} \equiv M_{1}(c, n),
$$

$|x-z| \leq|x-u|+\left|u-a_{u}\right|+\left|a_{u}-z\right| \leq 3 r_{v}$, and $d\left(\Delta_{u}\right) \geq b\left(\Delta_{u}\right) \geq r_{v} / c$. Since $t_{v}=8 r_{v}$, these estimates imply (iii) with $C_{1}=16 C_{0}\left(1+3 c M_{1}\right)$.

Case 2. $r_{u} \leq c s\left(a_{u}\right)$. By 3.9 we can choose a $c$-special simplex $\Delta_{u}$ for $\left(A, a_{u}, c s\left(a_{u}\right)\right)$. Since $s\left(a_{u}\right) \leq t_{u}$, we have

$$
\left\|S_{u}-f\right\|_{\Delta_{u}^{0}} \leq C_{0}\left\|S_{u}\right\| \varepsilon t_{u}
$$

by $(4.1)$.

We next show that

$$
s\left(a_{u}\right) \leq 8 r_{v}, \Delta_{u}^{0} \subset A\left(a_{v}, 8 c r_{v}\right) .
$$

Let $w \in \Delta_{u}^{0}$. If $a_{u} \neq a_{v}$, then $s\left(a_{u}\right) \leq\left|a_{u}-a_{v}\right| \leq 3 r_{v}$ by (i), and

$$
\left|w-a_{v}\right| \leq c s\left(a_{u}\right)+\left|a_{u}-a_{v}\right| \leq 6 c r_{v} .
$$

If $a_{u}=a_{v}$, then $s\left(a_{u}\right)=s\left(a_{v}\right) \leq 8 r_{v}$, proving (4.2).

Since $t_{v}=8 r_{v}$, it follows from (4.2) that $t_{u} \leq t_{v}$ and $\left\|S_{v}-f\right\|_{\Delta_{u}^{0}} \leq 16 C_{0}\left(\left\|S_{v}\right\| \vee\right.$ $\left.\left\|S_{u}\right\|\right) \varepsilon r_{v}$. As in Case 1, we choose a vertex $z$ of $\Delta_{u}$ and apply 3.11. For each $x \in Q$ we have

$$
|x-z| \leq d(Q)+r_{u}+c s\left(a_{u}\right) \leq 3 c s\left(a_{u}\right)
$$

and $d\left(\Delta_{u}\right) \geq b\left(\Delta_{u}\right) \geq s\left(a_{u}\right)$. Thus we obtain (iii) with $C_{1}=16 C_{0}\left(1+3 c M_{1}\right)$.

We next prove (iv). If $s\left(a_{u}\right) \geq 8 r_{u}$, then $a_{u}=a_{v}$ as above. This implies that $t_{u}=s\left(a_{u}\right)=s\left(a_{v}\right) \leq t_{v}$. If $s\left(a_{u}\right)<8 r_{u}$, then by (i) and (ii), we have $t_{u}=8 r_{u} \leq$ $16 r_{v} \leq 2 t_{v}$. 
To prove the inclusion of balls in (iv), let $x \in \bar{B}\left(a_{u}, t_{u}\right)$. Then

$$
\left|x-a_{v}\right| \leq\left|x-a_{u}\right|+\left|a_{u}-a_{v}\right| \leq t_{u}+6 r_{v} \leq 2 t_{v}+\frac{3}{4} t_{v} \leq 3 t_{v} .
$$

This completes the proof of (iv).

To prove (v), we apply 3.6 with $X=A\left(a_{u}, t_{u}\right)$. Since $t_{u} \geq s\left(a_{u}\right)$, we have $t_{u} \leq d(X)$, and by (iv), also $t_{v} \leq 2 t_{u} \leq 2 d(X)$. Assuming that $c \geq 3$, these and (iv) imply that the similarities $S_{u}$ and $S_{v}$ satisfy the approximation conditions of 3.6 in $X$, with $\varepsilon$ replaced by $2 C_{0} \varepsilon$, and (v) follows.

We have thus completed the proof of Fact 1.

Fact 2. There is a number $\delta_{2}(c, n)>0$ such that if $\varepsilon \leq \delta_{2}$ and $\Delta \in W$, then $F \mid \Delta$ is sense-preserving and $L$-bilipschitz with

$$
\frac{\left\|S_{v}\right\|}{1+C_{2} \varepsilon} \leq L \leq\left\|S_{v}\right\|\left(1+C_{2} \varepsilon\right)
$$

for all vertices $v \in \Delta$.

Proof. Let $Q \in K$ be the cube containing $\Delta$ and let $v \in Q \cap K^{0}$ be such that $r_{v}=\left|v-a_{v}\right|=d(v, A)$ is maximal. From Fact 1(iii) and from the construction of $F$ it follows that

$$
\left\|S_{v}-F\right\|_{\Delta} \leq C_{1} r_{v} \varepsilon\left\|S_{v}\right\|
$$

Here

$$
r_{v} \leq d(Q, A)+d(Q) \leq 4 d(Q)=8 b(\Delta) \sqrt{2 n},
$$

and thus $\left\|S_{v}-F\right\|_{\Delta} \leq \alpha b(\Delta) /(n+1)$, where

$$
\alpha=8 C_{1} \sqrt{2 n}(n+1) \varepsilon \leq \frac{1}{2}
$$

if $\varepsilon \leq \delta_{2}(c, n)=\left(16 \sqrt{2 n}(n+1) C_{1}\right)^{-1}$.

The claim now follows from [Vä, 2.7] for this particular vertex $v$. Also, $F \mid \Delta$ is $L$-bilipschitz with $L$ satisfying

$$
\left\|S_{v}\right\| /(1+2 \alpha) \leq L \leq\left\|S_{v}\right\|(1+2 \alpha) .
$$

Finally, from 3.6 the claim follows for all vertices of $\Delta$.

Finally, we show that $F$ can be well approximated by similarities in all balls. Our main theorem then follows from 3.7. The most important case is dealt with in Fact 3 below, and the rest are postponed into Fact 4 because of many cases and subcases that complicate the proof.

Fact 3. For each $a \in A$ and $r>0$, there is a sense-preserving similarity $S$ of $\mathbf{R}^{n}$ such that $S a=f(a)$ and

$$
\left\|S^{-1} F-\mathrm{id}\right\|_{\bar{B}(a, r)} \leq C_{3} \varepsilon r .
$$

Proof. If $s(a)>0$, then $F \mid K^{0}$ agrees with a similarity $S$ in $\bar{B}(a, s(a) / 8)$. This implies that $F=T$ in $\bar{B}(a, s(a) / 16)$. We may thus assume that $s(a) \leq 16 r$.

By 3.5 there is a special similarity $S$ for $(f, a, 20 \mathrm{cr})$. The map $S$ is sensepreserving and $S a=f(a)$. Let $x \in \bar{B}(a, r)$. We show that $|S x-F(x)| \leq C_{3} \varepsilon\|S\| r$ for $x \in \bar{B}(a, r)$, which implies that $S$ is the required similarity. Since

$$
\|S-f\|_{A(a, 20 c r)} \leq 20 C_{0} \varepsilon\|S\| r
$$

with $C_{0}=4 c^{2} c_{n}$ as before, we may assume that $x \in G=\mathbf{R}^{n} \backslash A$. 
Let $Q \in K$ be a cube containing $x$ and let $v \in Q \cap K^{0}$. It suffices to find an estimate $|S v-F(v)| \leq C_{3} \varepsilon\|S\| r$. We have $F(v)=S_{v} v$ and

$$
\left\|S_{v}-f\right\|_{A\left(a_{v}, c t_{v}\right)} \leq C_{0} \varepsilon t_{v}\left\|S_{v}\right\|
$$

with $t_{v}=s\left(a_{v}\right) \vee 8 r_{v}$. Since $c t_{v} \geq c s\left(a_{v}\right)$, there is a $c$-special simplex $\Delta_{v}$ for $\left(A, a_{v}, c t_{v}\right)$. As $d(Q) \leq d(Q, A) \leq r$, we have

$$
r_{v} \leq|v-a| \leq|v-x|+|x-a| \leq 2 r
$$

If $a_{v} \neq a$, this implies that $s\left(a_{v}\right) \leq\left|a_{v}-a\right| \leq 4 r$. If $a_{v}=a$, then $s\left(a_{v}\right)=s(a) \leq 16 r$. Hence

$$
t_{v} \leq 16 r \vee 8 r_{v}=16 r
$$

and thus $\Delta_{v}^{0} \subset A(a, 20 \mathrm{cr})$. This implies that

$$
\left\|S_{v}-S\right\|_{\Delta_{v}^{0}} \leq 20 C_{0} \varepsilon\|S\| r+C_{0} \varepsilon\left\|S_{v}\right\| t_{v} \leq 68 c C_{0} \varepsilon r\|S\|,
$$

since we show in the lemma below that $t_{v}\left\|S_{v}\right\| \leq 48 \mathrm{cr}\|S\|$. Fix a point $z \in \Delta_{v}^{0}$. Then

$$
|F(v)-S v| \leq 136 c C_{0} \varepsilon r\|S\|\left(1+M_{1}|v-z| / d\left(\Delta_{v}\right)\right),
$$

where $M_{1}=M_{1}(c, n)$ is the constant from the proof of Fact 1, and $|v-z| \leq r_{v}+$ $c t_{v} \leq 9 c t_{v} / 8, d\left(\Delta_{v}\right) \geq b\left(\Delta_{v}\right) \geq t_{v}$. Thus we obtain the required inequality with $C_{3}=136 c C_{0}\left(1+2 c M_{1}\right)$.

Lemma. Using the notation above, we have $t_{v}\left\|S_{v}\right\| \leq 48 \mathrm{cr}\|S\|$.

Proof. We have $t_{v} \leq 16 r$ from above. Choose an integrer $N$ such that $2^{N} t_{v} \geq 24 \mathrm{cr}$ and $2^{N-1} t_{v}<24 \mathrm{cr}$. Let $S_{k}$ be a special similarity for $\left(f, a_{v}, 2^{k} c t_{v}\right)$ so that $S_{0}=\bar{S}_{v}$ and $S_{N}=S$, and let $\Delta_{k}$ be a special simplex for $A\left(a_{v}, 2^{k} c t_{v}\right)$. Then $d\left(\Delta_{k}\right) \geq b\left(\Delta_{k}\right) \geq 2^{k} t_{v}$, and we can use 3.6 with $r=2^{k} t_{v}$ and $\varepsilon \mapsto 2 C_{0} \varepsilon$. In the last step we also use

$$
\bar{B}(a, 20 c r) \subset \bar{B}\left(a_{v}, 24 c r\right) \subset \bar{B}\left(a_{v}, 2^{N} t_{v}\right) .
$$

This implies that $\left\|S_{k}\right\| \leq\left(1+16 C_{0} \varepsilon\right)\left\|S_{k+1}\right\| \leq 2\left\|S_{k+1}\right\|$ under the requirement $\varepsilon \leq 1 / 16 C_{0}$. It follows that $\left\|S_{v}\right\| \leq 2^{N}\|S\| \leq 48 c\left(r / t_{v}\right)\|S\|$, which proves the lemma.

Fact 4. The corresponding result for Fact 3 is true for balls $\bar{B}(x, r)$ centered outside $A$.

Proof. Let $x \in \mathbf{R}^{n} \backslash A$ and $r>0$. The proof breaks up into several cases. All details are straightforward but rather long, cf. Fact 3, and therefore we omit most technicalities. As in the proof of Fact 3, the main idea in most cases is to find a suitable ball $\bar{B}\left(a_{v}, R\right) \supset \bar{B}(x, r)$ so that the special similarity for $\left(f, a_{v}, R\right)$ is the required one.

Case 1. The ball $\bar{B}(x, r)$ does not contain any vertices of the triangulation.

Subcase 1a. The ball $\bar{B}(x, r)$ is contained in some simplex $\Delta$ of the triangulation. In this case the extension $F$ is the convex combination of similarities $S_{v}$ for $v \in \Delta^{0}$. The claim follows from Facts 1 and 2 .

Subcase 1b. The ball $\bar{B}(x, r)$ is not included in a single simplex. In this case $\bar{B}(x, r)$ is contained in a finite union of adjacent Whitney cubes, whose number is bounded by a fixed constant depending on $n$. It follows from Facts 1 and 2 that the required similarity can be any of the similarities $S_{v}$, where $v$ is a vertex of a simplex containing $x$.

Case 2. The ball $\bar{B}(x, r)$ contains vertices of the triangulation. Choose a vertex $v \in K^{0} \cap \bar{B}(x, r)$ such that $d(v, A)$ is maximal. Excluding the trivial case (i) below, 
we claim that a special similarity $S$ for $\left(f, a_{v}, R\right)$ is the required one for a suitable radius $R$.

Subcase $2 \mathrm{a}(\mathrm{i}) . s\left(a_{v}\right)>0,\left|x-a_{v}\right| \leq s\left(a_{v}\right) / 16$, and $r \leq s\left(a_{v}\right) / 16$. In this case all the approximating similarities $S_{u}$ used to define $F(u)$ in $\bar{B}(x, r) \cap K^{0}$ coincide. Thus $F$ itself is a similarity in $\bar{B}(x, r)$.

Subcase 2a(ii). $s\left(a_{v}\right)>0,\left|x-a_{v}\right| \leq s\left(a_{v}\right) / 16$, and $r \geq s\left(a_{v}\right) / 16$. In this case

$$
\bar{B}(x, r) \subset \bar{B}\left(a_{v}, 2 r\right) \subset \bar{B}\left(a_{v}, 16 c r\right) .
$$

Since $16 c r \geq c s\left(a_{v}\right)$, we can use an approximating similarity $S$ for $\left(f, a_{v}, 16 c r\right)$.

Subcase 2a(iii). $s\left(a_{v}\right)>0,\left|x-a_{v}\right|>s\left(a_{v}\right) / 16$, and $r \geq r_{v} / 2$. In this case $\left|x-a_{v}\right| \leq|x-v|+\left|v-a_{v}\right| \leq r+r_{v} \leq 3 r$, and thus

$$
\bar{B}(x, r) \subset \bar{B}\left(a_{v}, 4 r\right) \subset \bar{B}\left(a_{v}, 128 c r\right) .
$$

Since $s\left(a_{v}\right)<16\left|x-a_{v}\right| \leq 64 r_{v}$, we have $128 c r \geq 64 c r_{v} \geq c s\left(a_{v}\right)$. In this case we use a special similarity $S$ for $\left(f, a_{v}, 128 c r\right)$.

Subcase $2 \mathrm{a}(\mathrm{iv}) . \quad s\left(a_{v}\right)>0,\left|x-a_{v}\right|>s\left(a_{v}\right) / 16$, and $r<r_{v} / 2$. In this case $\left|x-a_{v}\right| \leq 3 r_{v} / 2$, so that

$$
\bar{B}(x, r) \subset \bar{B}\left(x, 2 r_{v}\right) \subset \bar{B}\left(a_{v}, 7 r_{v} / 2\right) \subset \bar{B}\left(a_{v}, 24 c r_{v}\right) .
$$

Since $s\left(a_{v}\right)<16\left|x-a_{v}\right| \leq 24 r_{v}$, we can use a special similarity $S$ for $\left(f, a_{v}, 24 c r_{v}\right)$.

Subcase $2 \mathrm{~b}(\mathrm{i}) . \quad s\left(a_{v}\right)=0$ and $r_{v} \leq 2 r$. Let $S$ be a special similarity for $\left(f, a_{v}, 22 c r\right)$. Then

$$
\|S-f\|_{A\left(a_{v}, 22 c r\right)} \leq 22 C_{0} \varepsilon\|S\| r .
$$

For $u \in \bar{B}(x, r) \cap K^{0}$ we must estimate $|S u-F(u)|=\left|S u-S_{u} u\right|$, where $S_{u}$ satisfies $\left\|S_{u}-f\right\|_{A\left(a_{u}, c t_{u}\right)} \leq C_{0}\left\|S_{u}\right\| t_{u}$. If $a_{u} \neq a_{v}$, then

$$
s\left(a_{u}\right) \leq\left|a_{u}-a_{v}\right| \leq r_{u}+2 r+r_{v} \leq 6 r,
$$

so that $t_{u}=s\left(a_{u}\right) \vee 8 r_{u} \leq 6 r \vee 8 r_{v} \leq 16 r$. This is obviously true also in the case $a_{u}=a_{v}$, since then $s\left(a_{u}\right)=s\left(a_{v}\right)=0$. Let $w$ be a vertex of a special simplex $\Delta_{u}$ for $\left(A, a_{u}, c t_{u}\right)$. Then

$$
\left|w-a_{v}\right| \leq c t_{u}+\left|a_{u}-a_{v}\right| \leq 16 c r+6 r \leq 22 c r .
$$

This implies that $\Delta_{u}^{0} \subset A\left(a_{v}, 22 c r\right)$, and we can use $\Delta_{u}$ for estimating $\left|S u-S_{u} u\right|$. The details are essentially similar to the proof of Fact 3 , and are therefore omitted.

Subcase $2 \mathrm{~b}(\mathrm{ii}) . s\left(a_{v}\right)=0$ and $r_{v} \geq 2 r$. This case is very similar to the previous one, but now we choose a similarity $S$ satisfying

$$
\|S-f\|_{A\left(a_{v}, 12 c r_{v}\right)} \leq 12 C_{0} \varepsilon\|S\| r_{v} .
$$

If $a_{u} \neq a_{v}$, then

$$
s\left(a_{u}\right) \leq\left|a_{u}-a_{v}\right| \leq r_{u}+2 r+r_{v} \leq 3 r_{v},
$$

so that $t_{u}=s\left(a_{u}\right) \vee 8 r_{u} \leq 3 r_{v} \vee 8 r_{u} \leq 8 r_{v}$, which is true also in the case $a_{u}=a_{v}$. Since $\bar{B}\left(a_{u}, c t_{u}\right) \subset \bar{B}\left(a_{v}, 12 c r_{v}\right)$ in this case, we can again proceed as in the proof of Fact 3 .

This completes the proof of our main theorem. 


\section{References}

[AT1] Alestalo, P., and D. A. Trotsenko: Plane sets allowing bilipschitz extensions. - Math. Scand. 105, 2009, 134-146.

[AT2] Alestalo, P., and D. A. Trotsenko: On mappings that are close to a similarity. - Math. Rep. (Bucur.) 15:4, 2013, 313-318.

[ATV1] Alestalo, P., D. A. Trotsenko, and J. VÄIsÄlä: Isometric approximation. - Israel J. Math. 125, 2001, 61-82.

[ATV2] Alestalo, P., D. A. Trotsenko, and J. VÄIsälä: Linear bilipschitz extension property. - Sibirsk. Mat. Zh. 44, 2003, 1226-1238. Translation in Sib. Math. J. 44, 2003, 959-968.

[AH] Astala, K., and J. Heinonen: On quasiconformal rigidity in space and plane. - Ann. Acad. Sci. Fenn. Ser. A I Math. 13, 1988, 81-92.

[DF] Damelin, S. B., and C. Fefferman: On extensions of $\varepsilon$ differomorphisms. - arXiv: $1505.06950 \mathrm{v} 1,2015$.

[Jo] John, F.: Rotation and strain. - Comm. Pure Appl. Math. 14, 1961, 391-413.

[MS] Martio, O., and J. Sarvas: Injectivity theorems in plane and space. - Ann. Acad. Sci. Fenn. Ser. A I Math. 4, 1978/1979, 383-401.

[Pa] Partanen, J.: Invariance theorems for the bilischitz and quasisymmetric extension properties. - Ann. Acad. Sci. Ser. A I Math. Diss. 80, 1991, 1-40.

[PV] PARtanen, J., and J. VÄIsÄLÄ: Extension of bilipschitz maps of compact polyhedra. Math. Scand. 72, 1993, 235-264.

[Re] Reshetnyak, Yu. G.: Stability theorems in geometry and analysis. - Springer, 1994, 235264.

[Tr1] Trotsenko, D. A.: Continuation from a domain and approximation of spatial quasiconformal mappings with a small distortion coefficient. - Soviet Math. Dokl. 27, 1983, 771-780.

[Tr2] Trotsenko, D. A.: Approximation by similarities of spatial mappings with bounded distortion. - Sib. Math. J. 27, 1986, 196-205.

[TrV] Trotsenko, D. A., and J. VÄIsÄlä: Upper sets and quasisymmetric maps. - Ann. Acad. Sci. Fenn. Math. 24, 1999, 465-488.

[TuV] TukiA, P., and J. VÄIsÄLÄ: Quasisymmetric embeddings of metric spaces. - Ann. Acad. Sci. Fenn. Math. 5, 1980, 97-114.

[Vä] VÄıs̈̈Lё, J.: Bilipschitz and quasisymmetric extension properties. - Ann. Acad. Sci. Fenn. Math. 11, 1986, 239-274.

Received 2 March 2016 • Accepted 13 March 2016 